\title{
Spectroscopy of kaonic atoms at DAFNE and J-PARC
}

J. Marton ${ }^{1, *}$, A. Amirkhan ${ }^{4}$, A. Baniahmad ${ }^{4}$, M. Bazzi ${ }^{2}$, G. Bellotti ${ }^{4}$, C. Berucci ${ }^{2}$, D. Bosnar ${ }^{5}$, M. Bragadireanu ${ }^{3}$, M. Cargnelli ${ }^{1}$, C. Curceanu ${ }^{2}$, A. Dawood Butt ${ }^{4}$, R. Del Grande ${ }^{2}$, L. Fabbietti ${ }^{6}$, C. Fiorini ${ }^{4}$, F. Ghio ${ }^{10}$, C. Guaraldo ${ }^{2}$, M. Iliescu ${ }^{2}$, M. Iwasaki ${ }^{8}$, P. Levi Sandri ${ }^{2}$, M. Miliucci ${ }^{2}$, P. Moskal $^{9}$, S. Niedźwiecki ${ }^{9}$, S. Okada ${ }^{8}$, D. Pietreanu ${ }^{2,4}$, K. Piscicchia ${ }^{2,10}$, H. Shi ${ }^{2}$, M. Silarski ${ }^{9}$, D. Sirghi ${ }^{2,3}$, F. Sirghi ${ }^{2,3}$, M. Skurzok ${ }^{9}$, A. Spallone ${ }^{2}$, H. Tatsuno ${ }^{11}$, O. Vazquez Doce ${ }^{2,9}$, E. Widmann ${ }^{1}$, and J. Zmeskal ${ }^{1}$

${ }^{1}$ Stefan-Meyer-Institut für Subatomare Physik, Vienna, Austria

${ }^{2}$ INFN, Laboratori Nazionali di Frascati, Frascati (Roma), Italy

${ }^{3}$ Horia Hulubei National Institute of Physics and Nuclear Engineering (IFIN-HH), Magurele, Romania

${ }^{4}$ Politecnico di Milano, Dipartimento di Elettronica, Informazione e Bioingegneria and INFN Sezione di Milano, Milano, Italy

${ }^{5}$ Department of Physics, Faculty of Science, University of Zagreb, Croatia

${ }^{6}$ Excellence Cluster Universe, Technische Universiät München, Garching, Germany

${ }^{7}$ INFN Sez. di Roma I and Inst. Superiore di Sanita, Roma, Italy

${ }^{8}$ RIKEN, Tokyo, Japan

${ }^{9}$ The M. Smoluchowski Institute of Physics, Jagiellonian University, Kraków, Poland

${ }^{10}$ Museo Storico della Fisica e Centro Studi e Ricerche "Enrico Fermi”, Roma, Italy

${ }^{11}$ Lund Univeristy, Lund, Sweden

\begin{abstract}
The interaction of antikaons $\left(\mathrm{K}^{-}\right)$with nucleons and nuclei in the low-energy regime represents a very active research field in hadron physics. A unique and rather direct experimental access to the antikaon-nucleon scattering lengths is provided by precision X-ray spectroscopy of transitions in low-lying states in the lightest kaonic atoms (i.e. kaonic hydrogen and deuterium). In the SIDDHARTA experiment at the electron-positron collider DAFNE of LNFINFN we measured the most precise values of the strong interaction observables in conic hydrogen. The strong interaction on the 1s ground state of the electromagnetically bound K-p atom causes an energy shift and broadening of the 1s state. SIDDHARTA will extend the spectroscopy to kaonic deuterium to get access to the antikaon-neutron interaction and thus the isospin dependent scattering lengths. At J-PARC a kaon beam is used in a complementary experiment with a different setup for spectroscopy of kaonic deuterium atoms. The talk will give an overview of the of the upcoming experiments SIDDHARTA and the complementary experiment at J-PARC.Furthermore, the implications of the experiments for the theory of low-energy strong interaction with strangeness will be discussed.
\end{abstract}

\section{Introduction}

In the low-energy domain the interaction of antikaons $\left(\mathrm{K}^{-}\right)$with nucleons and nuclei numerous studies in experiment and theory were performed (for reviews see [1]). From the

\footnotetext{
*e-mail: johann.marton@oeaw.ac.at
} 
spectroscopy of higher $\mathrm{Z}$ kaonic atoms the potential of the $\bar{K} N$ interaction could be determined but it turns out that one has to take corrections in these multi-nucleon systems into account. The experiments with kaonic hydrogen and deuterium are of special interest these systems give a rather straightforward access to the $\mathrm{K}^{-}$interaction on the two isospin channels of the nucleon. In the $\bar{K} N$ interaction in the kaonic atoms the sub-threshold resonance $\Lambda(1405)$ has a strong impact. It influences strongly the hadronic shift and width of the $1 \mathrm{~s}$ groundstate at threshold. On the other hand it is still in discussion whether the $\Lambda(1405)$ is a one-pole or two-pole resonance [2]. The elementary case of the antikaon interaction with the nucleons give information about $\Lambda(1405)$ and it can be studied with kaonic hydrogen X-ray spectroscopy. In the past the results were puzzling, but could solved by an experiment at KEK [3]. The interaction of $\mathrm{K}^{-}(\bar{K})$ with nucleons in the two isospin states can be precisely determined by X-ray spectroscopy of the simplest kaonic atoms, i.e. kaonic hydrogen and kaonic deuterium. In these exotic atoms the strong interaction leads to an energy shift $\epsilon_{1 s}$ from the calculated electromagnetic value (i.e. without strong interaction) and a broadened width $\Gamma_{1 s}$ of the $1 \mathrm{~s}$ ground state. By measuring the X-ray transitions to the $1 \mathrm{~s}$ state $\epsilon_{1 s}$ and $\Gamma_{1 s}$ can be determined. Recently advances in the theoretical description of the $\bar{K} N$ interaction on the basis of field theory were made. The measured hadronic shift and width of kaonic hydrogen are used as anchor points for these calculations. In order to determine the isospinseparated scattering length for the isospin $\mathrm{I}=0$ scattering length $\mathrm{a}_{0}$ scattering length and the $\mathrm{I}=1$ scattering length $\mathrm{a}_{1}$, respectively.

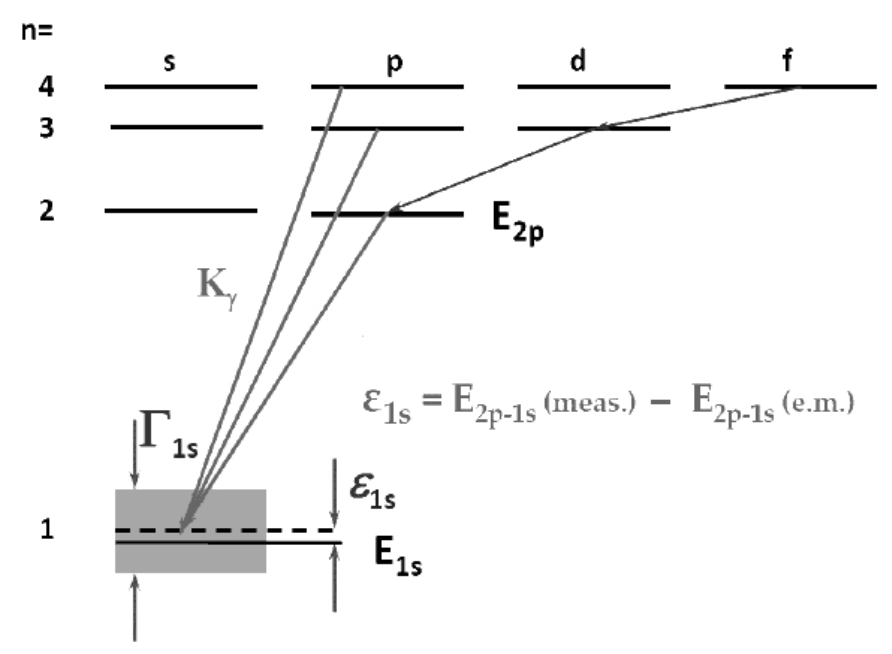

Figure 1. Transitions to the $1 \mathrm{~s}$ ground state in kaonic hydrogen. Only the $1 \mathrm{~s}$ state is measurably influenced by the strong antikaon-nucleon interaction. The calculated electromagnetic transition energy is about $6.5 \mathrm{keV}$ which can be precisely measured with SDDs.

The lightest kaonic atom is the $\mathrm{K}^{-} \mathrm{p}$ atom in which the principal interaction is the electromagnetic interaction accompanied by the strong interaction of the kaon with the proton which is measurable by X-ray spectroscopy of the radiative transitions from the p states $(2 \mathrm{p}$, $3 \mathrm{p}, \ldots$ for $\mathrm{n}=2,3, \ldots$.$) to the 1 \mathrm{~s}$ ground state ( $\mathrm{K}$ transitions). The energy shift $\epsilon_{1 s}$ is calculated by Equ.1, $\epsilon_{1 s}$ is given by the difference between the measured $\mathrm{E}_{n p \rightarrow 1 s}^{\text {meas. }}$ and the calculated electromagnetic transition energy $\mathrm{E}_{n p \rightarrow 1 s}^{e m}$. 


$$
\epsilon_{1 s}=E_{n p \rightarrow 1 s}^{\text {meas. }}-E_{n p \rightarrow 1 s}^{e m} .
$$

With the following improved Equ.2 [4] the scattering $\mathrm{K}^{-}$-p length $\mathrm{a}_{p}$ can be calculated taking corrections ito account

$$
\epsilon_{1 s}+\frac{i}{2} \Gamma_{1 s}=2 \alpha^{3} \mu_{c}^{2} a_{p}\left(1-2 \alpha \mu_{c}(\ln \alpha-1) a_{p}\right)
$$

Equ.3 gives the relation of this scattering length $\mathrm{a}_{p}$ to the isospin-dependent scattering lengths $\mathrm{a}_{0}$ (isospin 0$)$ and $\mathrm{a}_{1}$ (isospin 1).

$$
a_{p}=\frac{1}{2}\left(a_{0}+a_{1}\right)
$$

The measurement of X-ray spectrum of kaonic hydrogen imposes a challenge due to the small amount of kaons to be stopped in a low-density hydrogen gas and X-ray yield. In the framework of SIDDHARTA the experimental studies on kaonic atoms at DAФNE were focused on the X-ray spectroscopy of kaonic hydrogen and helium isotopes taking advantage of the ideal conditions of this $\Phi$-factory: Low-momentum $(127 \mathrm{MeV} / \mathrm{c}) \mathrm{K}^{-}$emitted back-toback in the $\Phi$ two-body decay (branching ratio of about $50 \%$. The SIDDHARTA experiment employed silicon drift detectors (SDDs) for X-ray spectroscopy. A triple coincidence of Xray and the back-to back emitted kaons from the $\Phi$ meson decay are possible due to the timing capability of SDDs. These successful experiments will be complemented by a first measurement of the even more challenging study of the X-ray spectrum of kaonic deuterium.

\section{SIDDHARTA results on light kaonic atoms}

SIDDHARTA succeeded in essential experimental results using the low-energy kaons from DAФNE and an array of SDD X-ray detectors.

\subsection{Kaonic helium isotopes}

SIDDARTA had many successes like the first measurement of the strong interaction in kaonic helium-3 [5] and the first measurement of kaonic helium-4 in a gas target [6].

\subsection{Kaonic hydrogen}

Certainly the highlight of SIDDHARTA was the up-tonow most precise measurement of the strong interaction in kaonic hydrogen. Out of these data $\epsilon_{1 s}$ and $\Gamma_{1 s}$ could be determined at unprecedented precision [7].

$$
\begin{aligned}
& \varepsilon_{1 s}=-283 \pm 36(\text { stat }) \pm 6(\text { syst }) \mathrm{eV} . \\
& \Gamma_{1 s}=541 \pm 89(\text { stat }) \pm 22(\text { syst }) \mathrm{eV} .
\end{aligned}
$$

Furthermore, the X-ray yields of the $\mathrm{K}$ transitions in kaonic hydogen were determined which is important for the understanding of the electromagnetic cascade processe in hadronic atoms [8].

SIDDHARTA also performed an exploratory X-ray study with a pure deuterium filling. An upper limit for the X-ray yield of the $\mathrm{K}$ lines could be extracted from the data: total yield $<0.0143, \mathrm{~K} \alpha$ yield $<0.0039$ [9]. However, $\epsilon_{1 s}$ and $\Gamma_{1 s}$ could not be determined due to the 
limited statistics and the background condition. Nevertheless, this study is important for the planning of the SIDDHARTA 2 experiment on kaonic deuterium.

To determined $a_{0}$ and $a_{1}$ it is necessary to measure strong interaction shift and width of kaonic deuterium. This is a far more challenging experimental issue. The kaonic deuterium case is still open and will be studied by SIDDHARTA-2 applying a significantly improved setup.

\section{Kaonic deuterium with SIDDHARTA2}

Experimentally the case of kaonic deuterium is still open and challenging due to the anticipated low X-ray yield ( $\sim 10 \%$ of the kaonic hydrogen yield) which demands highly efficient $\mathrm{X}$-ray detection and the expected larger hadronic width [10-14] which requests largely improved background suppression. For the Monte Carlo simulation of the kaonic deuterium $\mathrm{X}$-ray spectrum a value of $800 \mathrm{eV}$ for shift and width were used according to theory.

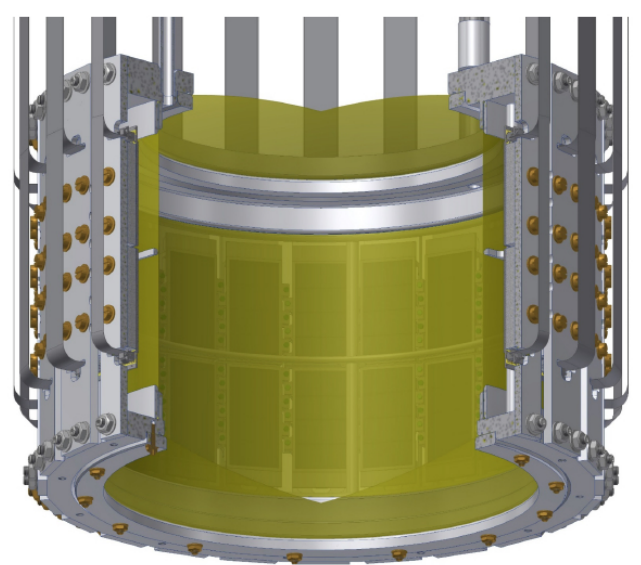

Figure 2. Design of the gas volume of SIDDHARTA 2 which provides the mandatory stabilty and the transmission for $\mathrm{X}$-rays. The volume will be surrounded by arrays of $\mathrm{X}$-ray detectors and shielding devices.

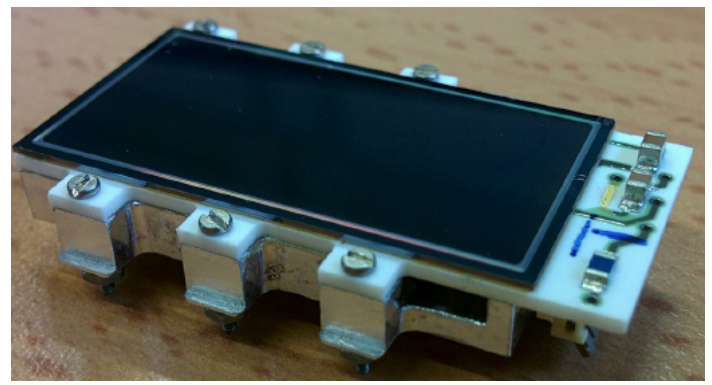

Figure 3. New SDD X-ray detectors for SIDDHARTA2 with a cell area of $64 \mathrm{~mm}^{2}$, total area of $512 \mathrm{~mm}^{2}$. The detectors will be cooled to about $170 \mathrm{~K}$ and have a drift time of smaller than $500 \mathrm{~ns}$.

A new experiment SIDDHARTA-2 [15-17] is planned which is based on a strongly improved apparatus. The improvements include an optimized geometry, deuterium gas density, 
discrimination of $\mathrm{K}^{+}$, active shielding and better SDD timing performance by cooling. According to Monte Carlo studies one expects an X-ray energy spectrum for an integrated luminosity of $800 \mathrm{pb}^{-1}$ shown in Fig. 4. For the hadronic shift and width one expects $30 \mathrm{eV}$ and $80 \mathrm{eV}$, respectively [18]. This experiment is planned for DAФNE and in a complementary experiment at J-PARC.

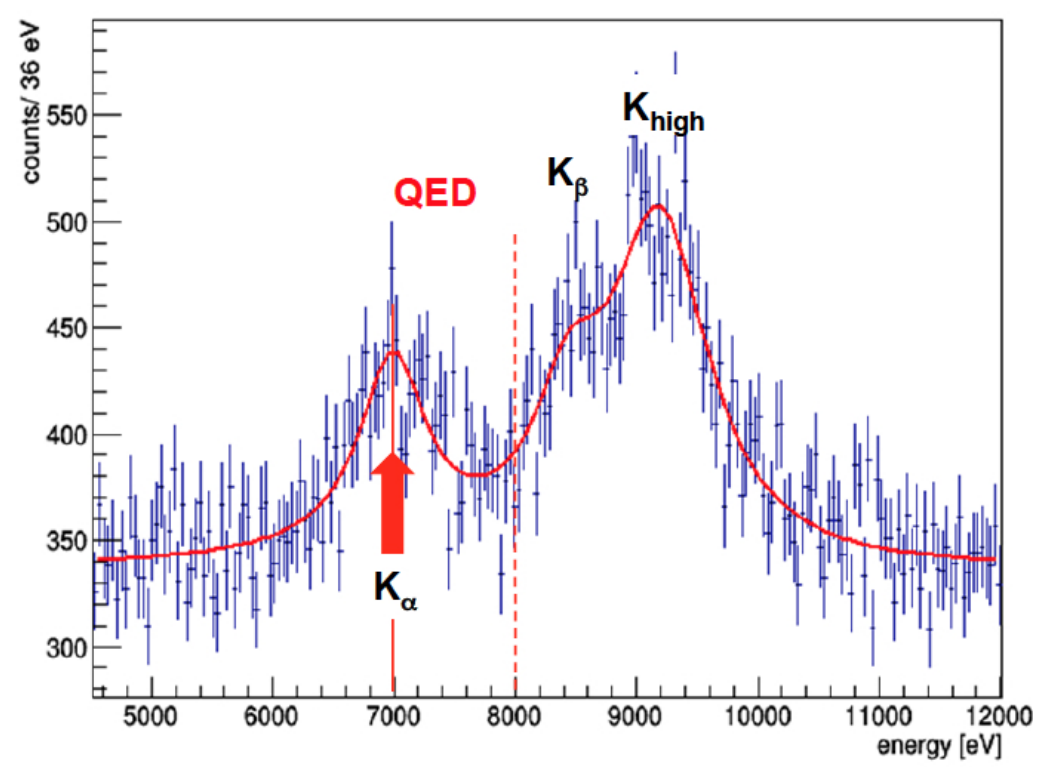

Figure 4. GEANT4 simulated kaonic deuterium X-ray spectrum (the pure electromagnetic $2 \mathrm{p}-1 \mathrm{~s}$ transition is indicated by an arrow) with a detector active area of $246 \mathrm{~cm}^{2}$ and assuming $\epsilon_{1} s=-800 \mathrm{eV}$ and $\Gamma_{1} s=800 \mathrm{eV}$ [19]. Withe a $\mathrm{K}_{\text {alpha }}$ yield of 0.1 percent the signal-to-background ratio is 1:4.

\section{Kaonic deuterium at J-PARC}

An experiment on kaonic deuterium [20,21] - complimentary to SIDDHARTA2 at LNF - will be performed at J-PARC (E57) using the K1.8BR kaon beam line and particle tracking by the central drift chamber (CDC) of the J-PARC-E15 experiment. The pure cryogenic deuterium target will be installed In the centre of the spectrometer and for the X-ry spectroscopy SDD derectors will surrounfd the target cell. The light-weight target cell is designed for a density of $5 \%$ of liquid hydrogen density for sufficienty kaon stopping. Since the momentum of the kaon beam is higher than in the case of SIDDHARTA 2 a massive degrader has to be installed in front of the deuterium target. The particle tracking with the large acceptence CDC will allow for an efficient background reduction. According to Monte Carlo simulations the hadronic shift and width of the $\mathrm{K}$ transitions in kaonic deuterium can be measured with a comparable precision like in SIDDHARTA2 - but with different systematics.

\section{Summary}

After the successful experiments of SIDDHARTA new experiments at LNF and J-PARC are in preparation for measuring the hadronic shift and width of kaonic deuterium using $\mathrm{X}$-ray spectroscopy. For the first time the interaction in both isospin channels will be 
quantitatively studied and the scattering lengths $\mathrm{a}_{0}$ and $\mathrm{a}_{1}$ will be extracted. These data will have tremendous impact on the theory of antikaon-nucleon interaction [22, 23], which might also have implications in the question about strangeness in the universe.

We thank C. Capoccia and G. Corradi, from LNF-INFN; and H. Schneider, L. Stohwasser, and D. Pristauz-Telsnigg from Stefan-Meyer-Institut, for their fundamental contribution in designing and building the SIDDHARTA setup. We thank as well the DAФNE staff for the excellent working conditions and permanent support. Part of this work was supported by the Austrian Science Fund (FWF): [P24756-N20]; Austrian Federal Ministry of Science and Research BMBWK 650962/0001 VI/2/2009; the Grantïn-Aid for Specially Promoted Research (20002003), MEXT, Japan; the Croatian Science Foundation, under project 1680; Minstero degli Affari Esteri e della Cooperazione Internazionale, Direzione Generale per la Promozione del Sistema Paese (MAECI), Strange Matter project; Polish National Science Center through grant No. UMO-2016/21/D/ST2/01155; Ministry of Science and Higher Education of Poland grant no 7150/E-338/M/2018.

\section{References}

[1] E. Friedman, A. Gal, Phys. Rep. 452, 89 (2007), C.J. Batty, E. Friedman, A.Gal, Phys. Rep. 287, 385 (1997)

[2] T. Hyodo and D. Jido, Prog. Part. Nucl.Phys. 67, 55 (2012); arXiv:1104.4474 [nucl-th]

[3] M. Iwasaki et al., Phys. Rev. Lett. 78, 3097 (1997), T. M. Ito et al., Phys. Rev. C 58, 2366 (1998)

[4] U.-G. Meißner, U. Raha, A. Rusetsky, Eur. Phys. J. C 47, 473 (2006)

[5] M. Bazzi et al. (SIDDHARTA Coll.), Phys. Lett. B 697, 199 (2011)

[6] M. Bazzi et al. (SIDDHARTA Coll.), Phys. Lett. B 681, 310 (2009); M. Bazzi et al., Phys. Lett. B 714, 40 (2012)

[7] M. Bazzi et al. (SIDDHARTA Coll.), Phys. Lett. B 704, 113 (2011), M. Bazzi et al. (SIDDHARTA Coll.), Nucl. Phys. A 881, 88 (2012)

[8] M. Bazzi et al., Nucl. Phys. A 954, 7 (2016)

[9] M. Bazzi et al. (SIDDHARTA Coll.), Nucl. Phys. A 907, 69 (2013)

[10] S.S. Kamalov, E. Oset, A. Ramos, Nucl. Phys. A 690, 494 (2001)

[11] A. Gal, Int. J. Mod. Phys. A 22, 226 (2007)

[12] M. Döring, U.-G. Meißner, Phys. Lett. B 704, 663 (2011)

[13] N.V. Shevchenko, Nucl. Phys. A 890-891, 50 (2012)

[14] T. Mizutani, C. Fayard, B. Saghai, K. Tsushima, Phys. Rev. C 87, 035201 (2013)

[15] C. Curceanu et al., Acta Phys. Pol. B 48, 1855 (2017)

[16] H. Shi et al., J. Phys.: Conf. Ser. 800, 012007 (2017)

[17] A. Scordo et al. (SIDDHARTA Coll.), EPJ Web of Conferences 181, 01004 (2018)

[18] D. Sirghi et al., Proc. BEACH 2018, to be published

[19] M. Cargnelli, private communication

[20] H. Tatsuno et al., EPJ Web of Conferences 130, 01018 (2016)

[21] J. Zmeskal et al., JPS Conf. Proc. 17, 071001 (2017)

[22] Y. Ikeda, T. Hyodo and W. Weise, Few-Body Syst 54, 1113 (2013)

[23] W. Weise, Hyp. Int. 233, 131 (2015) 